\section{Fatores que influenciaram o processo de formulação de políticas de recursos humanos em saúde no Brasil e em Portugal: estudo de caso múltiplo}

\author{
Factors that influence human resources for \\ health policy formulation: a multiple case \\ study in Brazil and Portugal
}

Ana Paula Cavalcante de Oliveira 1 Mario Roberto Dal Poz 2

Isabel Craveiro 1

Mariana Gabriel 3

Gilles Dussault 1

doi: 10.1590/0102-311X00220416

\title{
Resumo
}

O estudo objetiva analisar o processo em que as políticas de recursos humanos em saúde (RHS), que visam melhorar a distribuição geográfica dos médicos, são (ou não) informadas por evidência científica no Brasil e em Portugal. Foi realizado um estudo de caso-múltiplo sobre o processo de decisão das políticas de RHS no Brasil e em Portugal. Para compor os estudos de caso, as políticas escolhidas foram o Programa Mais Médicos (PMM) e a estratégia de contratação de médicos estrangeiros por acordos bilaterais para o trabalho no Serviço Nacional de Saúde (SNS) português. Foram entrevistados 27 atores-chave no processo de formulação das politicas em análise nos seguintes tópicos: fatores que influenciaram a formulação, atores que eram esperados ganhar ou perder, evidências científicas e os dados disponiveis utilizados para a formulação, entre outros. Os fatores mais evidentes identificados nas entrevistas como sendo influenciadores do PMM foram: Instituições; Fatores Externos (eleições presidenciais); Interesses de grupos (por exemplo, associações de profissionais médicos), governos (brasileiro e cubano), organização internacional e sociedade civil; e Ideias (evidência científica). Os fatores mais listados em Portugal foram: Instituições e Interesses dos governos (português e envolvidos nos acordos bilaterais), sociedade civil e grupos (associações de profissionais médicos). Ao contrário do que se verificou no estudo de caso do Brasil, em que reconhecidamente a evidência teve um papel importante na formulação da política em análise, em Portugal a evidência científica não foi identificada como contributo para a formulação da intervenção em estudo.

Recursos Humanos em Saúde; Políticas Públicas de Saúde; Formulação de Políticas; Área Carente de Assistência Médica; Acesso aos Serviços de Saúde

\author{
Correspondência \\ A. P. C. Oliveira \\ Global Health and Tropical Medicine, Instituto de Higiene e \\ Medicina Tropical, Universidade Nova de Lisboa. \\ Rua da Junqueira 100, Lisboa - 1349-008, Portugal. \\ anapaula_olive@hotmail.com \\ 1 Instituto de Higiene e Medicina Tropical, Universidade Nova \\ de Lisboa, Lisboa, Portugal. \\ 2 Instituto de Medicina Social, Universidade do Estado do \\ Rio de Janeiro, Rio de Janeiro, Brasil. \\ 3 Faculdade de Odontologia, Universidade de São Paulo, \\ São Paulo, Brasil.
}




\section{Introdução}

A formulação de políticas de saúde informadas pela melhor evidência de pesquisa disponível é uma abordagem que auxilia os formuladores a tomarem decisões mais assertivas 1. Caracteriza-se pela avaliação da evidência de forma transparente de investigações relevantes, visando garantir que outros possam examinar que evidência foi utilizada de forma apropriada 1 . As potenciais vantagens são a estruturação da formulação das políticas, ao invés do direcionamento pelos principais atores 2,3; auxiliar o público em geral e tomadores de decisão a compreender uma problemática e escolher uma direção política mais adequada; atingir uma maior transparência na utilização do dinheiro público; e reduzir ou evitar o risco de insucesso da política 3 . Em contrapartida, a não utilização de evidência pode conduzir à subutilização de intervenções eficazes, ao uso incorreto de intervenções adequadas e ao uso excessivo de intervenções não comprovadas 4,5. Esse cenário pode criar consequências graves aos utilizadores dos serviços como, por exemplo, comprometer o acesso aos cuidados de saúde adequados, aumentar as iniquidades em saúde 1 e levar ao uso ineficiente de recursos limitados para a prestação de cuidados de saúde 6 .

Apesar dos avanços registrados nos últimos vinte anos nos processos de utilização de evidência, nem todas as lições aprendidas, na área de políticas concernentes à prática clínica, podem necessariamente ser aplicadas a outras áreas. Alguns trabalhos de revisão investigaram os fatores que impedem ou facilitam o uso de evidência científica para informar as decisões políticas em áreas legislativas e administrativas no setor da saúde 7,8 . Poucos estudos examinam o uso da evidência científica no processo de formulação de política no contexto de influências concorrentes 9 . O uso de evidência na formulação de políticas poderia ser potencializado se os pesquisadores conhecessem as influências concorrentes no processo político, formassem parcerias com os tomadores de decisões, desafiassem os incentivos dentro das instituições de pesquisa e se envolvessem em debates públicos sobre as problemáticas em pesquisa 9 .

Este estudo objetiva analisar o processo em que as políticas de recursos humanos em saúde (RHS), que visam melhorar a distribuição geográfica dos médicos, são (ou não) informadas por evidência científica no Brasil e em Portugal. Mais especificamente, identificar e analisar: os fatores que influenciam a formulação de políticas de RHS, que pretendem melhorar a distribuição geográfica de médicos; os esforços para o uso de evidência na formulação destas políticas; e as estratégias utilizadas para potencializar a formulação de políticas de RHS informadas por evidências científicas.

\section{Método}

Estudo de caso-múltiplo sobre o processo de decisão das políticas de RHS no Brasil e em Portugal desenvolvido em três fases: (I) e (II) análise dos contextos políticos de RHS dos dois países 10,11; (III) o foco, é explorado o processo da formulação da política e os fatores que a influenciaram na perspectiva dos atores envolvidos.

As políticas foram escolhidas por apresentarem o mesmo objetivo, isto é, reduzir a distribuição assimétrica de médicos nas zonas com necessidades de serviços de saúde não atendidas (rural, remota ou carente) e serem executadas majoritariamente na atenção primária à saúde (APS) (Tabela 1).

\section{Coleta de dados e amostragem}

Entre novembro de 2015 e abril de 2016 foram conduzidas entrevistas semiestruturadas de forma presencial, pela autora A.P.C.O., com atores-chave no processo de formulação das políticas em análise. Foram entrevistados 27 atores de 35 pedidos enviados ("amostragem bola de neve”). Após o máximo de três tentativas de contato, não foram entrevistados oito atores - seis brasileiros e dois portugueses. Os atores entrevistados encontravam-se em um dos três grupos (Tabela 2).

Foi utilizado um roteiro semiestruturado, com os seguintes tópicos: a história da política, fatores que influenciaram a formulação, atores que eram esperados ganhar ou perder com a implantação, evidências científicas e dados disponíveis usados para a formulação e mecanismos promovidos pelo Ministério da Saúde para facilitar o uso e acesso aos resultados de pesquisas. As entrevistas foram cen- 


\section{Tabela 1}

Descrição das estratégias políticas analisadas para compor os estudos de caso.

Estratégias analisadas (país)

I. Programa Mais Médicos (Brasil)

II. Contratação de médicos estrangeiros por acordos bilaterais (Portugal)

\section{Descrição das estratégias}

Lançado em julho de 2013 com a Medida Provisória no 621 e regulamentado pela Lei no 12.871 de 22 de outubro de 2013, a qual estabeleceu o programa como uma política de Estado. Tem como finalidade formar médicos e levá-los para localidades e regiões onde existe escassez ou ausência de profissionais. Para atingir os seus objetivos o programa foi estruturado em três grandes eixos de ação:

- Eixo de qualificação da estrutura da atenção básica: melhoria na infraestrutura da Rede de Atenção à Saúde (RAS), com foco nas unidades básicas de saúde (UBS);

- Eixo de provimento emergencial, intitulado "Projeto Mais Médicos para o Brasil" (PMMB), que atua na provisão emergencial de médicos em áreas vulneráveis. O recrutamento dos médicos ocorre de forma individual provenientes do Brasil e de outros países (com a relação de médicos por mil habitantes maiores do que a do Brasil) e por meio de acordo bilateral estabelecido com o governo cubano ${ }^{34}$. Os participantes do programa recebem uma bolsa mensal e ajuda de custos para a instalação, as quais são mais elevadas para os participantes que se deslocam para zonas mais remotas; e

- Eixo da formação médica: mudanças na graduação, na residência médica e na formação de especialistas 35 .

Formulada para dar resposta à escassez de médicos de família em Portugal, principalmente em áreas carentes. O primeiro acordo bilateral começou em 2008 e foi realizado entre os governos português e uruguaio, contratando 15 médicos para atuarem no Instituto Nacional de Emergência Médica (INEM). Em 2009, outro acordo foi assinado com Cuba, com renovação em 2012 e 2014, perfazendo cerca de 200 médicos, especificamente para desenvolver atividades em centros de saúde nas regiões do Algarve, Alentejo e Lisboa/Vale do Tejo (LVT). Em 2011, iniciaram os acordos bilaterais com a Colômbia (120 médicos) e a Costa Rica (12 médicos). Todos os médicos estrangeiros contratados nesse âmbito passaram por um processo de seleção em seu país. A Faculdade de Medicina do Porto (FMP) realizou exames escritos aos candidatos. Antes de chegarem a Portugal os selecionados participaram de um curso de Português. Depois da validação dos diplomas pela FMP e do exame de Português, registaram-se na Ordem dos Médicos. Foi organizada uma breve formação sobre o Serviço Nacional de Saúde (SNS) português e houve um período de duas semanas para a adaptação ao serviço.

tradas nas percepções e experiências dos entrevistados de forma individual na formulação da política, e não expressam a instituição ou o cargo que o ator ocupava.

\section{Análise das entrevistas}

As entrevistas foram gravadas e transcritas. Como framework para a análise dos dados coletados foi utilizado o quadro conceitual 3Is (Interesses, Ideias e Instituições), incluindo os Fatores Externos (3Is+E) (Figura 1).

O quadro baseia-se em três fatores que a literatura da ciência política usa para explicar o processo de desenvolvimento das políticas públicas 12,13. Tem como principio que o desenvolvimento e as 


\section{Tabela 2}

Grupos dos atores entrevistados no Brasil e em Portugal.

\begin{tabular}{|c|c|c|}
\hline Grupo dos atores entrevistados & Brasil & Portugal \\
\hline $\begin{array}{l}\text { Formuladores de política de nível central (FP) e técnicos (TMS) envolvidos na formulação da } \\
\text { política em análise }\end{array}$ & $\begin{array}{l}4 \text { atores, sendo } 3 \mathrm{FP} \\
\text { e } 1 \mathrm{TMS}\end{array}$ & $\begin{array}{l}8 \text { atores, sendo } 7 \text { FP } \\
\text { e } 1 \text { TMS }\end{array}$ \\
\hline $\begin{array}{l}\text { Pesquisadores }(P) \text { citados pelos formuladores de política ou referenciados nos documentos } \\
\text { relevantes }\end{array}$ & 5 & 3 \\
\hline $\begin{array}{l}\text { Grupo de interesses - outros atores interessados de organizações internacionais (OI), entidades } \\
\text { médicas (EM), conselhos - por exemplo, Conselho Nacional de Saúde, Conselho Nacional de } \\
\text { Secretários de Estado (CS) }\end{array}$ & $\begin{array}{l}5 \text { atores, sendo } 2 \text { OI, } \\
\qquad 1 \mathrm{EM}, 2 \mathrm{CS}\end{array}$ & 2 EM \\
\hline
\end{tabular}

Figura 1

Quadro conceitual: 3Is (Interesses, Ideias e Instituições) + Fatores Externos (3Is+E).

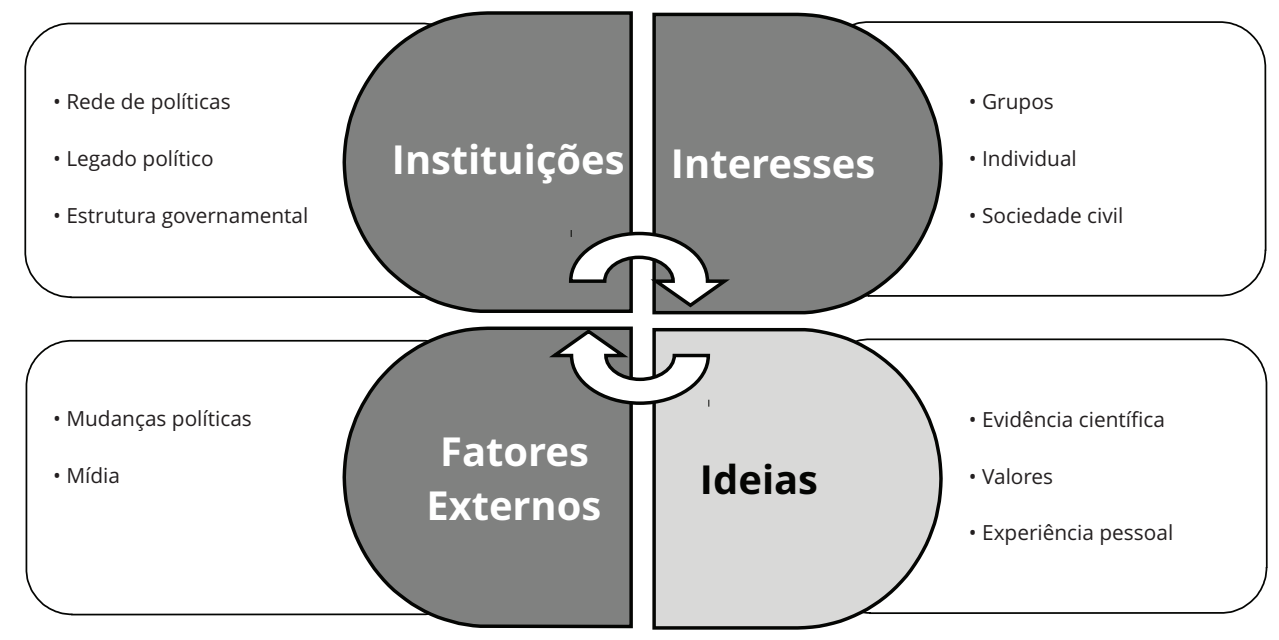

Fonte: adaptado de Lavis et al. 12 .

escolhas da política podem ser influenciados pelos Interesses e Ideias dos atores e a configuração das Instituições 12,13 (Tabela 3).

A análise temática dos dados ocorreu dedutivamente, por meio de categorias e subcategorias prédeterminadas propostas no quadro conceitual e indutivamente com subcategorias que emergiram a partir da leitura das entrevistas transcritas.

\section{Considerações éticas}

Pesquisa aprovada pelo Comitê de Ética em Pesquisa do Instituto de Medicina Social da Universidade do Estado do Rio de Janeiro - Brasil (CAAE: 49733215.4.0000.5260) e pelo Conselho de Ética do Instituto de Higiene e Medicina Tropical da Universidade Nova de Lisboa - Portugal. 


\section{Tabela 3}

Fatores influenciadores das políticas públicas.

\begin{tabular}{|c|c|}
\hline Fatores influenciadores & Considerações \\
\hline Instituições & $\begin{array}{l}\text { As Instituições são as regras formais e informais, normas, precedentes e fatores organizacionais que estruturam } \\
\text { o comportamento político 12. O fator Instituições é composto por diversos subfatores entre os quais está o legado } \\
\text { político, que refere-se a atributos de políticas anteriores que podem moldar o processo político por intermédio da } \\
\text { criação de incentivos e dando a alguns atores o acesso a mais ou menos recursos em comparação com outros; e a } \\
\text { estrutura governamental e características do processo de formulação de política (como, por exemplo, o tempo de } \\
\text { resposta a um problema e o nível de aprovação exigido para uma política num dado país) que podem estruturar o } \\
\text { comportamento político 13,36. }\end{array}$ \\
\hline Interesses & $\begin{array}{l}\text { Os Interesses estão relacionados com as agendas das várias partes interessadas - grupos sociais, funcionários } \\
\text { públicos, funcionários eleitos, pesquisadores e empreendedores de políticas 12,13. O fator Interesses (agendas de } \\
\text { várias partes interessadas) é o desejo de influenciar o processo de formulação de políticas para alcançar seus fins, de } \\
\text { forma a impulsionar ou impedir o desenvolvimento de escolhas políticas 12,13. Esse fator expressa as percepções dos } \\
\text { atores políticos sobre quem se beneficia e quem perde com o resultado de uma determinada política e quanto 12,13. }\end{array}$ \\
\hline Ideias & $\begin{array}{l}\text { As Ideias podem influenciar a forma como os atores definem o problema e como percebem as diferentes opções } \\
\text { políticas como eficazes, viáveis e aceitáveis. Este fator inclui a investigação e também outros tipos de informações e } \\
\text { valores dos formuladores de políticas, assessores políticos e outras partes interessadas } 12,13 \text {. }\end{array}$ \\
\hline Fatores Externos & $\begin{array}{l}\text { O quadro referencial também considera Fatores Externos, os quais podem propiciar oportunidades para introduzir } \\
\text { alterações no contexto da formulação de uma política } 12,13 \text {. }\end{array}$ \\
\hline
\end{tabular}

\section{Resultados e discussão}

Para possibilitar a comparação, os resultados estão organizados em subseções em torno dos objetivos de investigação.

\section{Fatores que influenciaram a formulação das estratégias estudadas}

Os fatores mais evidentes identificados nas entrevistas como sendo influenciadores do Programa Mais Médicos (PMM) foram: (Ia) Instituições, com as subcategorias referentes à estrutura governamental e ao legado político; (Ib) Fatores Externos (eleições presidenciais); (Ic) Interesses de grupos (prefeitos e associações de profissionais médicos), governos (brasileiro e cubano), organização internacional (Organização Pan-Americana da Saúde - OPAS) e sociedade civil; e Ideias (evidência científica). Os fatores mais listados nas entrevistas em Portugal foram: (IIa) Instituições, legados políticos e a estrutura governamental; e (IIb) Interesses dos governos (português e envolvidos nos acordos bilaterais), sociedade civil e grupos (associações de médicos). Ver Tabela 4 para exemplos de citações dos entrevistados.

\section{- $\quad$ la - Fatores Institucionais do PMM (Brasil)}

Relativamente à estrutura governamental, os formuladores entrevistados relataram que as dificuldades internas do Ministério da Saúde, a burocracia e a centralização do processo de formulação e execução do PMM, num país da dimensão do Brasil, dificultaram o processo de formulação da política (BR_FP1). Segundo os formuladores, o Ministério da Saúde esteve no papel de formulador, coordenador, implementador do PMM e condutor das transformações no processo de formação dos profissionais médicos, juntamente com o Ministério da Educação (MEC) e a Casa Civil. Foram também envolvidos o Ministério das Relações Exteriores, Defesa, Receita Federal, Polícia Federal, o Ministério do Trabalho e Emprego, e o Banco do Brasil (BR_TMS/BR_FP1). O papel do Ministério da Saúde como agente normativo e formulador de políticas foi questionado por entrevistados do grupo de interesses, segundo os quais o Ministério da Saúde com o PMM passou a desenvolver um papel 


\section{Tabela 4}

Exemplos de citações por fatores e subfatores por política analisada.

\begin{tabular}{|c|c|c|c|}
\hline Estratégia & Fator & Subfatores & Citação \\
\hline \multirow[t]{9}{*}{ PMM } & Instituições & $\begin{array}{l}\text { Estrutura } \\
\text { governamental } \\
\text { - burocracia e a } \\
\text { centralização do } \\
\text { processo }\end{array}$ & $\begin{array}{l}\text { "O Ministério da Saúde é duro, é uma burocracia pesada, é um processo difícil, é um país com muitas } \\
\text { regras, com muitas dificuldades, com muitas situações, é evidente de que o Brasil e que no Ministério } \\
\text { um processo centralizado num país desse tamanho não é uma operação fácil" (BR_FP1). } \\
\text { "Há no Ministério da Saúde nos últimos anos uma equivocada recentralização das ações de saúde. o } \\
\text { Ministério passou de um papel normativo e formulador de política a um papel muito operativo e de } \\
\text { ação muito verticalizada junto aos territórios municipais e estaduais. Eu acho isso crítico, acho que } \\
\text { isso desobedece à arquitetura do SUS..." (BR_CS1). }\end{array}$ \\
\hline & Instituições & $\begin{array}{l}\text { Legados políticos } \\
\text { - preceito da } \\
\text { constituição } \\
\text { e programas } \\
\text { anteriores }\end{array}$ & $\begin{array}{c}\text { "E ao mesmo tempo a gente também resgatou o preceito constitucional, onde no seu artigo } 200 \text { a } \\
\text { Constituição fala que é prorrogativa do SUS a ordenação da formação de RH, então se deu uma } \\
\text { dimensão de que seria necessário não só programas, por exemplo, de atrair profissionais para um } \\
\text { determinado local e sim de pensar desde a formação, pensar em educação permanente, pensar as } \\
\text { várias dimensões" (BR_FP2). }\end{array}$ \\
\hline & & & $\begin{array}{l}\text { "A gente tem o entendimento que, por exemplo, não seria viável a implantação tão rápida do PMM, } \\
\text { que em } 9 \text { meses já tinha } 14 \text { mil médicos participando do programa, se não fosse a experiência que } \\
\text { a gente já tinha com o PROVAB. Então essa experiência do Ministério da Saúde de fazer chamada } \\
\text { nacional de médicos, de alocar os médicos em municípios, de fazer os pagamentos destes médicos, } \\
\text { supervisionar estes médicos e acompanhar o desenvolvimento das atividades" (BR_FP2). }\end{array}$ \\
\hline & $\begin{array}{l}\text { Fatores } \\
\text { Externos }\end{array}$ & $\begin{array}{l}\text { Eleições } \\
\text { presidenciais }\end{array}$ & $\begin{array}{l}\text { “...parecia, por todas as evidências existentes, que o principal problema era a necessidade do governo } \\
\text { na etapa pré-eleitoral de ter um programa estrela, um programa conhecido na área da saúde } \\
\text { (...) Estava muito sincronizado com o calendário eleitoral, o qual pode ser lógico, pois muita coisa } \\
\text { acontece, por razões políticas, este caso tinha uma relação política forte” (BR_OI). }\end{array}$ \\
\hline & Interesses & $\begin{array}{l}\text { Atores que } \\
\text { ganharam - } \\
\text { governos (Cuba e }\end{array}$ & $\begin{array}{c}\text { "...a presidenta quis bancar o programa, tinha população pedindo por mais acesso nas jornadas, } \\
\text { então teve essa janela de oportunidade, os prefeitos pedindo, a presidenta apoiando e o Brasil com } \\
\text { recursos" (BR_TMS). }\end{array}$ \\
\hline & & $\begin{array}{l}\text { Brasil), prefeitos } \\
\text { de municípios } \\
\text { atendidos pelo }\end{array}$ & $\begin{array}{c}\text { "Referindo-se às marchas, '...aquilo ali acelerou, fez a presidência falar: olha, aquela proposta que } \\
\text { vocês estavam construindo está pronta ou não está pronta?'. Está pronta. Então vamos lançar" } \\
\text { (BR_FP3). }\end{array}$ \\
\hline & & $\begin{array}{l}\text { PMM, sociedade civil } \\
\text { e OPAS }\end{array}$ & $\begin{array}{c}\text { "Um ator que ganhou muito foi a OPAS, que ganhou } 50 \text { milhões de dólares e uma grande relevância } \\
\text { com o governo" (BR_OI). }\end{array}$ \\
\hline & Interesses & $\begin{array}{l}\text { Potencial perdedor } \\
\text { com a formulação } \\
\text { do programa - } \\
\text { classe médica }\end{array}$ & $\begin{array}{c}\text { "Eu acho que não perdeu ninguém, eu não vejo. Eu vi que a AMB e o CFM, inicialmente, fizeram uma } \\
\text { oposição forte (...) mas depois descobriram que na verdade estes médicos estavam proibidos de } \\
\text { atuar fora deste programa, portanto, não eram e nunca teriam sido uma concorrência (...) Eu acho } \\
\text { que o programa provou que dá um certo ganho a todo mundo" (BR_OI). }\end{array}$ \\
\hline & Ideias & $\begin{array}{l}\text { Evidência científica - } \\
\text { uso na formulação }\end{array}$ & $\begin{array}{l}\text { “Desde } 2011 \text { que estou aqui no ministério, a gente usa muita evidência para tomar decisão. Por } \\
\text { exemplo, para a gente poder discutir a PNAB a própria presidenta pediu artigos. Ela virou e falou: } \\
\text { olha, eu quero alguns artigos que mostrem o acerto da PNAB, se é saúde da família mesmo? Qual é o } \\
\text { impacto? Como deve ser a melhor maneira de investir dinheiro na atenção básica?..." (BR_FP3). } \\
\text { “...meados de maio de 2013, a presidenta chamou uma reunião com as entidades médicas para } \\
\text { ouvir estas entidades sobre o problema que os prefeitos estavam trazendo muito fortemente e ouvir } \\
\text { destas entidades quais as alternativas que elas viam (...) Então se criaram canais de discussão desta } \\
\text { temática. A questão era a dificuldade de serem canais propositivos, porque era não à abertura de } \\
\text { novas escolas médicas, porque era não à vinda de médicos de outros países...” (BR_FP2). } \\
\text { “Um dizia falta médicos, outro dizia tem médicos, mas estão mal distribuídos. Que na verdade as } \\
\text { duas coisas são verdade, só que um fazia ênfase em um e outro fazia ênfase em outro (...). Mas foi } \\
\text { um diálogo de surdos, não foi uma discussão. O governo já tinha a decisão firme de trazer médicos } \\
\text { de Cuba, já tinha muito antes, pelo menos um ano antes... E a AMB e o CFM no mundo inteiro têm a } \\
\text { posição contrária a importar médicos de outro país” (BR_Ol1). }\end{array}$ \\
\hline
\end{tabular}

(continua) 
Tabela 4 (continuação)

\begin{tabular}{|c|c|c|c|}
\hline Estratégia & Fator & Subfatores & Citação \\
\hline & Ideias & $\begin{array}{l}\text { Evidência científica } \\
\text { - mecanismos para } \\
\text { promover o uso de } \\
\text { evidência científica }\end{array}$ & $\begin{array}{l}\text { “Esses dados serviram para apoiar, eles já eram trabalhados antes independentes, quero dizer } \\
\text { esse sentimento, essa evidência já existia. Então a política se apoiou nessas evidências que já } \\
\text { existiam para ser implementada (...) Os estudos foram financiados pelo Ministério da Saúde e pelo } \\
\text { Observatório, no nosso caso, e via OPAS" (BR_P4). } \\
\text { "Então variam os mecanismos. Quando a gente está querendo uma coisa mais rápida e que o } \\
\text { colaborador possa apresentar resultados intermediários, justamente para poder elucidar e trazer } \\
\text { evidência para a política, normalmente a gente financia diretamente. Porque se a gente faz por edital, } \\
\text { começa a pesquisa hoje e daqui a dois anos, três anos você tem algum resultado. E ainda depois o } \\
\text { cara vai fazer o artigo e ele não libera antes de fazer o artigo. Quando sai o artigo você já deixou de } \\
\text { ser gestor. Quando a gente está querendo fazer para alimentar o processo decisório a gente financia } \\
\text { diretamente" (BR_FP3). }\end{array}$ \\
\hline & Ideias & $\begin{array}{c}\text { Evidência científica - } \\
\text { motivos do uso }\end{array}$ & $\begin{array}{l}\text { “O segundo relatório era muito interessante porque ele era usado tanto pelo CFM, pelas entidades } \\
\text { para comprovarem que não faltam médicos no Brasil, como ele era usado para dizer que 'olha como } \\
\text { a distribuição de médicos é irregular e faltam médicos'. Então, se você olhar a demografia médica } \\
\text { ela serve de base tanto para posicionamentos das entidades médicas contrárias ao Mais Médicos } \\
\text { quanto ela compõe os PowerPoints e materiais de divulgação, dizendo que a demografia médica } \\
\text { comprova, até traz evidência de que faltam médicos e que precisa. Então, aspectos que possam ser } \\
\text { negativos para um ou outros propósitos políticos, eles são selecionados dentro da produção e da } \\
\text { evidência científica" (BR_P1). } \\
\text { "Eu acho que promove, como todo gestor promove. O gestor não decide a priori em função da } \\
\text { evidência científica, ele decide em função da pressão política ou da demanda política que está lá. } \\
\text { Depois ele aprimora a resposta a partir da evidência científica, mas esta questão é muito mais } \\
\text { disparada pela perceção às vezes meio difusa de uma certa realidade e que depois é aprimorada } \\
\text { através de evidência" (BR_P2). }\end{array}$ \\
\hline \multirow[t]{4}{*}{$\begin{array}{l}\text { Acordos } \\
\text { bilaterais }\end{array}$} & Instituições & $\begin{array}{l}\text { Legados políticos - } \\
\text { experiência passada } \\
\text { de acordo bilateral }\end{array}$ & $\begin{array}{l}\text { "Foi calculado em } 2008 \text { que precisávamos aproximadamente de } 7 \text { a } 10 \text { anos para ter o número } \\
\text { de médicos suficiente para que todo o cidadão português tivesse um médico de família. Isso era } \\
\text { especialmente difícil em algumas regiões do país em que as pessoas não queriam vir. E dado já ter } \\
\text { tido alguma experiência internacional nisso. Porque em } 2006 \text { quando eu precisava de médicos que } \\
\text { trabalhassem só em emergência médica (...) e não arranjava em Portugal. Porque em Portugal o } \\
\text { mercado médico em termos de oferta e procura está invertido. Então, houve uma oportunidade } \\
\text { de fazer um acordo com o Uruguai para trazer médicos para trabalhar em emergência médica" } \\
\text { (PT_FP3). }\end{array}$ \\
\hline & Instituições & $\begin{array}{c}\text { Estrutura } \\
\text { governamental } \\
\text { - centralização } \\
\text { do processo de } \\
\text { formulação }\end{array}$ & $\begin{array}{l}\text { “...era eu e duas secretárias. Portanto, quando eu saí não houve ninguém que ocupasse esta função. } \\
\text { Portanto, eu tinha que fazer tudo, contratava Instituto Camões, para ensinar a história portuguesa. } \\
\text { Contratava professores de português... a política foi definida toda no ministério e, portanto, como o } \\
\text { meu trabalho respondia diretamente ao ministro, era muito mais fácil, não é?” (PT_FP3). }\end{array}$ \\
\hline & Interesses & $\begin{array}{c}\text { Interesses - } \\
\text { necessidade política } \\
\text { e da população }\end{array}$ & $\begin{array}{l}\text { "Política porque qualquer governo quer dizer que deu médico para toda a gente. Depois havia que } \\
\text { haver alternativas, já disse, só havia duas [referindo-se a aumentar a lista de usuários por médico } \\
\text { ou a contratação de médicos estrangeiros] (...) porque não havia médicos" (PT_FP3). } \\
\text { "Em 2008, estávamos confrontados com isso, nós tínhamos feito o que era estrategicamente } \\
\text { adequado para resolver a carência de médicos, mas estávamos com carência de médicos porque as } \\
\text { medidas estratégias iriam demorar tempo para produzir efeito" (PT_FP2). } \\
\text { "O objetivo de Portugal era sempre o mesmo. O que davam ao país tinha que ser de acordo com o } \\
\text { que os países queriam ou precisavam" (PT_F3). }\end{array}$ \\
\hline & Interesses & $\begin{array}{l}\text { Certa resistência } \\
\text { à formulação da } \\
\text { estratégia - classe } \\
\quad \text { médica }\end{array}$ & $\begin{array}{c}\text { “Eu não consegui identificar ninguém que pudesse perder... Há sempre uma certa pressãozinha } \\
\text { corporativa, mas eu acho que toda a gente ganhou com isso... E baseado sempre num número geral, } \\
\text { que é Portugal, tem médicos que cheguem na comparação internacional. Talvez tenha. Eu não vou } \\
\text { questionar isso... Portugal pode ter, em Odemira não há. Eu abri concurso e ninguém concorreu (...) } \\
\text { eu acho que a minha obrigação enquanto decisor político é encontrar um médico que queira ir para } \\
\text { Odemira" (PT_FP2). }\end{array}$ \\
\hline
\end{tabular}

(continua) 
Tabela 4 (continuação)

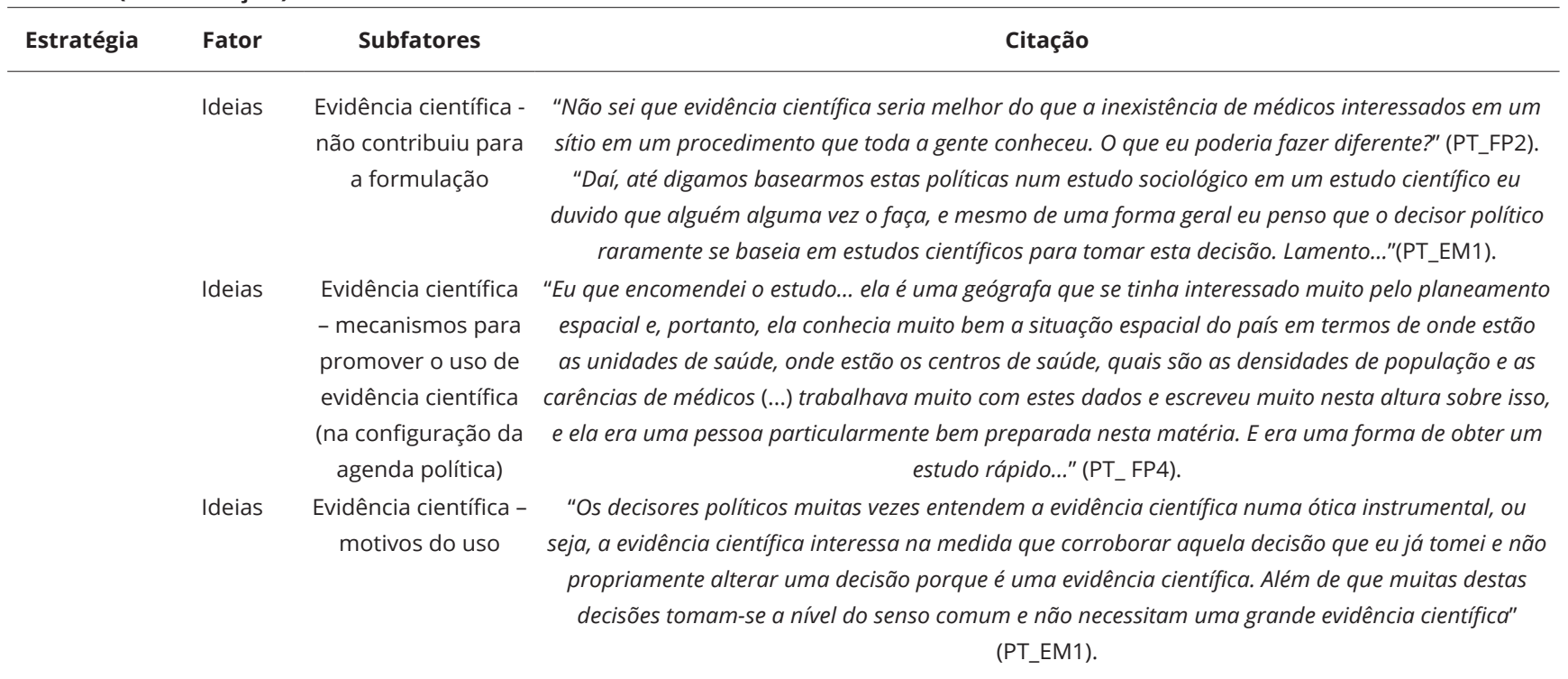

AMB: Associação Médica Brasileira; CFM: Conselho Federal de Medicina; OPAS: Organização Pan-Americana da Saúde; PMM: Programa Mais Médicos; PNAB: Política Nacional de Atenção Básica; PROVAB: Programa de Valorização do Profissional da Atenção Básica; RH: recursos humanos; SUS: Sistema Único de Saúde.

operacional, indo contra a organização do Sistema Único de Saúde (SUS) e indicando uma possível recentralização das ações de saúde (BR_CS1).

Os formuladores identificaram que legados políticos impulsionaram e moldaram o processo da formulação e também atenuaram as dificuldades. Foi utilizado o preceito do artigo 200 da Constituição Federal, segundo o qual o SUS tem a competência de ordenar a formação de RHS. Essa competência fundamentou intervenções adicionais aos programas de formação e educação permanente, ou seja, foram ampliadas as áreas de intervenção política utilizadas na atuação da problemática de distribuição de médicos no país (BR_FP2).

Os formuladores e atores do grupo de interesses também realçaram a importância de programas anteriores ao PMM que objetivavam corrigir a má distribuição dos médicos. O Programa de Interiorização do SUS (PISUS) e o Programa de Interiorização do Trabalho em Saúde (PITS) ajudaram no entendimento de que intervenções isoladas tendem a não gerar uma sustentabilidade a longo prazo, sendo necessária a utilização de múltiplas intervenções em diferentes áreas políticas para que o objetivo e a sustentabilidade sejam alcançados. As experiências desses programas foram curtas em duração, reduzidas em número de profissionais abrangidos e não alteraram questões cruciais do processo da formação médica (BR_CS1/BR_FP1/BR_FP2).

Programas mais recentes fortaleceram a capacidade institucional do Ministério da Saúde, sendo fundamentais para desenvolver condições de formular e implementar o PMM. Essas experiências anteriores possibilitaram maior agilidade e dimensão ao processo de implantação do PMM (BR_FP2/ BR_TMS).

\section{- Ib - Fatores Externos ao PMM (Brasil)}

Quanto aos Fatores Externos, quase todos os atores entrevistados do grupo de interesses mencionaram a eleição presidencial como um fator influenciador no processo de formulação do PMM, e indicaram a necessidade de haver uma formulação rápida de um programa que pudesse ser atribuído como marca de governo na área da saúde. 


\section{- Ic - Fator Interesses do PMM (Brasil)}

Para os três grupos de entrevistados, os atores que mais ganharam com o programa foram: governos (Cuba e Brasil); prefeitos de municípios atendidos pelo PMM; sociedade civil; e a OPAS. A classe médica foi identificada como um potencial perdedor com a formulação do PMM.

Segundo os formuladores, o marco inicial do PMM aconteceu com a entrada de uma nova gestão no Ministério da Saúde, que em seus primeiros meses de atuação em 2011 organizou em Brasília um seminário nacional sobre escassez, provimento e fixação de profissionais de saúde em áreas remotas e de maior vulnerabilidade. Adicionalmente, o Plano Plurianual e a Política Nacional de Atenção Básica (PNAB), centrais na organização do SUS, apresentavam a falta de profissionais médicos dentre as principais dificuldades para qualificar e expandir a APS, demonstrando-se que este tema seria uma prioridade para a política de saúde naquele momento.

De acordo com os formuladores, o PMM foi uma decisão política da Presidência da República, que em fevereiro de 2012 incumbiu o Ministério da Saúde de formular um programa que pudesse aumentar a oferta e a qualidade dos profissionais na APS. O tema foi discutido durante um ano e cinco meses, tempo em que informações foram pesquisadas para estruturar o diagnóstico, propor intervenções e identificar lugares prioritários para a atuação. A partir de abril de 2013, começou-se a antever a possibilidade de cooperação com Cuba (BR_TMS).

Ainda segundo os formuladores, no início de 2013 o Programa de Valorização do Profissional da Atenção Básica (PROVAB) ocupava 3.500 vagas, entretanto, as demandas das prefeituras eram de 13 mil médicos. Naquele momento, um dos atores citados em quase todas as entrevistas como um dos mais importantes no período, a Frente Nacional de Prefeitos (FNP), começou a campanha chamada Cadê o Médico?. A FNP valorizava as iniciativas anteriores como o Fundo de Financiamento ao Estudante do Ensino Superior (FIES) e o PROVAB, mas indicava que ainda eram necessárias mais ações. Para a advocacia de uma intervenção nessa problemática, os prefeitos utilizaram evidências não sistemáticas ou estruturadas (BR_FP1).

Os protestos da sociedade civil, de junho e julho de 2013, iniciados com a "marcha dos 20 centavos", foram identificados como catalisadores da política pela maioria dos entrevistados 14. A saúde estava entre os temas em debate e dentre as reivindicações estavam a melhoria da qualidade e ampliação do acesso aos serviços (BR_FP3). Aquele momento foi identificado, por um dos atores do grupo de interesse, também como uma janela de oportunidade para a formulação de uma política de RHS no Brasil que se fechou (BR_CS1).

O governo cubano e a OPAS foram também identificados como ganhadores por intermédio da formulação do programa. Para os atores do grupo de interesses o PMM foi concebido inicialmente como um acordo político entre o governo de Cuba e o governo do Brasil com a participação da OPAS, apresentando sobretudo características de cooperação econômica (BR_OI). Ocorreu um aumento da relevância da OPAS na interação com o governo brasileiro (BR_OI).

Por fim, verificou-se alguma relutância em identificar os atores que poderiam perder com a formulação do PMM. Os formuladores afirmaram que até um certo momento as entidades médicas, nomeadamente a Federação Nacional dos Médicos, Associação Brasileira de Medicina e Conselho Federal de Medicina (CFM), apoiaram as iniciativas como o PROVAB. Contudo, embora o PMM não fosse identificado pelos formuladores e alguns atores do grupo de interesses como prejudicial à empregabilidade ou à renda dos médicos brasileiros (referindo-se especificamente ao eixo emergencial de contratação de médicos estrangeiros), na percepção destes atores o grupo de entidades médicas mostrou-se resistente e receoso de que iriam perder com a implantação do PMM (BR_FP1). Também, foi apontado por um ator do grupo de interesses um ganho final inesperado para os médicos brasileiros, que querem ter esses médicos para cobrir lugares não cobertos (BR_OI).

A pressão exercida pelas entidades médicas contra a formulação do PMM levou à alteração de suas ações. No início de sua formulação incluía o "segundo ciclo na medicina", que consistia na extensão do curso de medicina por mais dois anos com um estágio na APS, mas devido a resistência, essa intervenção foi alterada. 


\section{- Ila - Fatores Institucionais dos acordos bilaterais (Portugal)}

Fator Institucional (legados políticos), a experiência passada do acordo bilateral desenvolvido com o governo uruguaio para a prestação de serviços de emergência médica foi atribuída por formuladores como um fator influenciador na formulação. A possibilidade desse acordo foi identificada pela troca de experiências entre formuladores de política de saúde de ambos os governos em um encontro íbero-americano, tendo o início de sua formulação ocorrido entre 2005 e 2006 e implantação em 2008. A contrapartida de Portugal nesse acordo era o treinamento de médicos-cirurgiões e enfermeiros em transplantes de fígado durante um ano no Hospital Curry Cabral em Lisboa e o apoio da equipe portuguesa nos primeiros transplantes realizados no Uruguai. Com base nessa experiência, o governo português encomendou em 2008 ao responsável pela execução do acordo bilateral com o governo uruguaio um plano de contratação de médicos estrangeiros (PT_FP3/PT_FP4).

Outro fator atribuído como facilitador por um formulador (estrutura governamental) foi o fato de o processo ter sido desenvolvido no Ministério da Saúde e concentrado em um único formulador com acesso direto ao Ministro (PT_FP3).

\section{- Ilb - Fator Interesses dos acordos bilaterais (Portugal)}

Para os entrevistados, os atores que mais ganharam com os acordos bilaterais foram os governos envolvidos e a sociedade civil. Um potencial "perdedor" identificado foi a classe médica representada pelas associações profissionais.

Relativamente ao Fator Interesses, a formulação foi identificada como indispensável devido à necessidade política e da população (PT_FP3). Quanto à necessidade da população, existia um grande número de usuários sem médico de família atribuído, sendo este fato uma barreira para desenvolver o programa de reforma da APS. Foram implantadas várias estratégias isoladas e ad hoc para atuar nessa problemática, como a implementação de uma quota de $25 \%$ de vagas de residência médica para médicos de família. Segundo os formuladores entrevistados, essas estratégias não apresentavam uma capacidade de resposta a curto prazo, o que exigiu uma intervenção que obtivesse uma resposta "mais imediatista” do que formar médicos (PT_FP2). Mas, segundo os atores interessados, os acordos bilaterais não tiveram expressão numérica e foram implementados para resolver problemas pontuais em regiões como o Alentejo e o Algarve (PT_EM1).

Conforme os formuladores, a opção da contratação de médicos da América Latina surgiu com a impossibilidade de contratar médicos de países europeus, que seria a opção inicial, devido à validação automática do diploma. Mas os salários em alguns desses países são iguais ou superiores aos de Portugal e o custo de vida mais baixo, como, por exemplo, na Polônia, e as condições oferecidas por outros países europeus com falta de médicos, como Inglaterra, Finlândia, Suécia, Noruega e Alemanha são mais atrativas (PT_FP2).

Todos os acordos bilaterais apresentavam uma contrapartida do governo português, com exceção da Costa Rica, onde a negociação foi feita com o Conselho de Medicina, que não a solicitou. A compensação de Cuba era financeira, um valor pago ao governo cubano para que os médicos selecionados com experiências em países da Comunidade dos Países da Língua Portuguesa (CPLP) prestassem cuidados de saúde em Portugal por três anos. Relativo à Colômbia, a contrapartida foi um acordo entre universidades onde estudantes desenvolveriam projetos de investigação, dissertações de mestrado e teses de doutoramento entre Portugal e Colômbia.

Segundo os formuladores, a Ordem dos Médicos ofereceu uma certa resistência à estratégia, embora os médicos portugueses fossem identificados como atores que não perderiam, na medida em que os acordos bilaterais previam que os profissionais trabalhassem no país pelo período de três anos, findos os quais estes teriam de retornar ao seu país. Na oposição política ao governo, o argumento utilizado era de que Portugal não teria uma falta de médicos e sim estes estariam mal distribuídos. Conforme indicado por um formulador, essa retórica impediu a ação política ao longo dos anos (PT_FP2). 


\section{Fator Ideias}

A problemática de distribuição geográfica de médicos não é enfrentada unicamente por estes países em estudo, esta vem sendo debatida mais enfaticamente nos últimos dez anos pela Academia. Embora exista um conjunto de possíveis estratégias identificadas com maior ou menor poder de evidência 15,16,17, não existe um modelo a ser seguido que possa ser implementado em qualquer país e que ofereça resolubilidade à problemática. Portanto, é necessário compreender as causas da problemática, características e necessidades do sistema e dos profissionais no país, para assim executar a escolha e ajustes necessários ao conjunto de estratégias a serem implementadas.

A utilização da evidência científica na formulação de políticas é um processo complexo, por vezes não sistemático e transparente, sendo influenciado por inúmeros fatores visto que a tomada de decisão não é um processo unicamente técnico, mas também inerente a um processo social 1,12. A complexidade desse processo, especificamente o da formulação de políticas de RHS, vem aumentando ao longo dos últimos anos devido ao número de atores envolvidos no processo e ao aumento da evidência científica, em volume e complexidade. Como foi possível verificar pelas entrevistas, a investigação não foi o único fator influenciador na formulação das políticas, sendo assim, é necessário o entendimento do processo de formulação como um todo para compreender quais as necessidades de pesquisa e em que momento é possível decorrer o seu uso.

$\mathrm{Na}$ análise dos esforços para potencializar o uso da evidência na formulação dessas estratégias, escolheu-se examinar a utilização da evidência científica no processo e também em que momento foi utilizada 18,19, com que finalidade 20,21, quais abordagens 22 e estratégias usadas para potencializar a formulação das políticas informadas por evidência científica.

\section{- Fator Ideias do PMM (Brasil)}

De acordo com os entrevistados, as evidências científicas geradas por resultados de pesquisas e outras evidências, como dados do CFM e do Ministério da Saúde, foram utilizadas no PMM com o intuito de informar e também opor-se à sua formulação $23,24,25,26,27$. No que se refere ao momento em que foi utilizada, os formuladores informaram que o uso da evidência científica nacional aconteceu na identificação do problema e suas causas, nas necessidades de ajustes das estratégias escolhidas para a implementação, e mais recentemente na avaliação do PMM. Além disso, para a identificação das possíveis soluções foram utilizadas a literatura internacional, revisões sistemáticas internacionais e outros estudos de estratégias usados em diferentes países 15,16,17. Igualmente, foi apontada a troca de experiências com formuladores de outros países, em reuniões da Organização Mundial da Saúde (OMS), relativamente a estratégias implantadas para corrigir essa problemática. Conforme identificado por um dos pesquisadores, nunca uma política gerou tanto investimento em pesquisa para a avaliação de um programa como o PMM (BR_P2).

Segundo formuladores e pesquisadores, o PMM gerou um debate entre as organizações médicas e o Ministério da Saúde. Segundo os formuladores, as organizações médicas se opuseram ao PMM em sua formulação e no estágio inicial de implementação, utilizando resultados de pesquisa 27 . Também utilizaram o argumento de que a falta de incentivos para os médicos trabalharem nesses locais era $o$ principal problema e não a sua escassez (BR_P4).

Segundo pesquisadores e formuladores, uma das abordagens que promoveu o uso da evidência científica foi o esforço para a coleta de evidência pelo usuário que busca a investigação para extrair informações e assim tomar decisões. Ou seja, esse tipo de esforço é apropriado em situações em que os potenciais utilizadores identificaram uma lacuna no conhecimento e querem resolver de uma forma oportuna 22. Segundo formuladores, pesquisadores e alguns atores do grupo de interesses, outra abordagem utilizada foi o esforço de intercâmbio, que ocorre quando produtores ou provedores de investigação desenvolvem parcerias significativas com um grupo de usuários que os ajudam a levantar questões relevantes e a respondê-las 22. A Secretaria de Gestão do Trabalho e da Educação na Saúde (SGTES) do Ministério da Saúde tem uma relação com um grupo de pesquisadores da Rede Observatórios de RHS no Brasil (ObservaRH), que produziram ao longo do tempo um conjunto de pesquisas, encomendadas e/ou financiadas pelo Ministério da Saúde, que serviram para informar a formulação do programa (BR_P4). 
Segundo os formuladores, um mecanismo encontrado para trazer agilidade ao processo de utilização dos resultados de investigação no Ministério da Saúde foi o financiamento direto com a apresentação de resultados intermediários pelo pesquisador. De acordo com o formulador, foi estimado que o mecanismo de financiamento tradicional do Ministério da Saúde (edital) requer em torno de dois a três anos para a obtenção dos resultados, mais o tempo da produção do artigo (BR_FP3).

Quanto à finalidade que a evidência científica foi utilizada, a principal discussão no campo é centrada em torno de três tipos de uso da investigação: instrumental, conceitual e simbólico 8,20. No caso específico do PMM não existe um consenso entre as partes interessadas sobre a finalidade da utilização da evidência gerada por pesquisas variando entre o uso conceitual, que envolve uma forma mais geral e indireta do uso da evidência; uso instrumental, que envolve a aplicação dos resultados de investigação de formas específicas e diretas; e o uso simbólico, em que a investigação é utilizada para validar e apoiar decisões previamente tomadas por outras razões 20,28.

Alguns atores do grupo de interesses e pesquisadores questionaram se, no caso específico do PMM, a evidência foi utilizada de forma adequada. Por exemplo, um dos pesquisadores mencionou que o relatório da demografia médica 27 foi utilizado tanto pelas entidades médicas contrárias ao PMM - para indicar que não faltavam médicos no Brasil - como pelos formuladores para apoiar a informação de falta de médicos. Entrevistados do grupo de pesquisadores e atores interessados do PMM indagaram se o volume anormal e desproporcional de investimentos em pesquisas, comparado com outros componentes do sistema de saúde, foi para uma validação do PMM (BR_P1).

Por fim, foram identificados dois determinantes que facilitaram o processo do uso da evidência, o reconhecimento da importância da utilização da evidência na formulação de políticas pelos atores envolvidos no processo, inclusive pelos formuladores, e a característica do processo de formulação da política no país, que inclui a existência de espaços de discussões.

A maioria dos entrevistados reconheceu a importância da utilização de evidências de pesquisa em todas as fases do processo de decisão política no Brasil e a cultura organizacional de seu uso. Um dos formuladores referiu que a evidência, seja de pesquisa nacional ou internacional, é utilizada para elucidar os debates políticos, atitude tomada pela Presidência da República, Casa Civil e Ministério da Saúde (BR_FP3). Contudo, alguns pesquisadores entrevistados reconheceram que a evidência não é o único fator influenciador da formulação da política, podendo não ser "prioritário" entre os fatores concorrentes. Sendo a evidência utilizada para o aprimoramento da resposta a uma problemática, que a princípio foi influenciada por outros fatores como a pressão ou a demanda política (BR_P2).

De acordo com os formuladores, existiram diversos espaços de discussão das possíveis soluções para a problemática de distribuição de médicos no país, como no Conselho Nacional de Saúde (CNS). As discussões nesses espaços sobre o PMM duraram aproximadamente quatro meses, antes da implantação do programa em julho de 2013 e durante o trâmite da lei da medida provisória (BR_FP2).

Segundo o grupo de interesses, essas interlocuções aconteceram de forma rápida e já em um momento de apresentação das possíveis intervenções e não na formulação. Foi uma etapa de discussão técnica com divergência de informações entre os atores. Membros do governo expuseram, apoiados por dados e estudos, uma falta de médicos nos municípios, e as associações médicas apresentaram, apoiados também em estudos encomendados, que o problema não era a quantidade de médicos e sim a distribuição destes e que, portanto, era um problema de melhoria do sistema de carreira (BR_OI1).

Em uma decisão política, mesmo entre especialistas, pode ocorrer a divergência de opiniões sobre a significância, a interpretação e a percepção de suficiência da evidência para a tomada de decisão, ou até, a utilização da mesma para defender diferentes soluções políticas para um problema. Nesse cenário, alguns grupos interessados, como os especialistas, podem exercer o poder e influenciar que tipo de evidência será utilizada, com que intuito e em que momento 29 . Segundo um dos pesquisadores entrevistados, o uso de evidência científica de institutos renomados no país pode dar uma forma de poder aos formuladores, uma espécie de poder criado, talvez, pela "questão da cientificidade", pela imparcialidade do pesquisador. Dessa forma, empoderar os cidadãos e outros atores para a utilização de evidência científica de forma a advogar uma estratégia e sua participação na discussão política pode contribuir para com a redução de seu uso incorreto (BR_P3). 


\section{- Fator Ideias dos acordos bilaterais (Portugal)}

A evidência científica não contribuiu para a formulação da estratégia de contratação de médicos estrangeiros em Portugal. Segundo os formuladores, para a elaboração dessa estratégia foi utilizada a troca de experiências com formuladores de outros países (PT_FP2).

A evidência científica teve seu papel na introdução da problemática na agenda governamental, contribuindo na identificação da problemática e no consequente levantamento da necessidade de formulação da estratégia em questão por meio de um estudo descritivo 30 e um quantitativo prospectivo de necessidade de médicos 31 . Esses estudos foram encomendados aos pesquisadores de forma direta pelo Ministério da Saúde 31 e pelo Ministério da Educação 30. Outros tipos de evidências como relatórios técnicos de informações extraídas das bases de dados elaboradas por grupos técnicos do Ministério da Saúde foram igualmente utilizados (PT_FP4).

A abordagem para promover o uso da evidência científica na configuração da agenda (inclusão da problemática de distribuição) foi o esforço para a coleta de evidência pelo usuário, o mesmo mecanismo utilizado no Brasil. O financiamento direto foi identificado em Portugal como forma para trazer agilidade ao processo de utilização dos resultados de investigação no Ministério da Saúde (PT_ FP4).

Os acordos bilaterais em Portugal geraram um debate e as organizações médicas se opuseram, utilizando resultados de pesquisa já em sua fase de implantação ${ }^{32}$. As organizações utilizaram o mesmo argumento usado pelas organizações médicas brasileiras - de que a falta de incentivos para os médicos trabalharem nos locais carentes era o principal problema e não a sua escassez (PT_EM1).

A maioria dos entrevistados reconheceu o potencial do uso de pesquisas na fase inicial do processo de decisão política (configuração da agenda política). Adicionalmente, alguns atores interessados questionaram se a evidência científica poderia ser utilizada de forma adequada ou somente para apoiar uma decisão previamente tomada (simbólica).

Dessa forma, a baixa prioridade que a principal fonte de apoio financeiro à investigação, a agência de financiamento pública nacional, oferece à pesquisa em serviços de saúde ou RHS 33 e, consequentemente, à limitação de estudos na área, somada ao não reconhecimento pelos atores da importância do uso da evidência em outras fases da política, pode ter refletido no uso da investigação centrada na identificação da problemática para a formulação dos acordos bilaterais em Portugal.

Este trabalho apresenta algumas limitações inerentes ao método de estudo de caso como a não possibilidade de generalização dos achados para outro contexto (validade externa). Para assegurar a credibilidade dos achados (validade interna), os passos metodológicos foram seguidos com rigor: roteiro de entrevista testado e triangulação das fontes de informação usando distintos procedimentos de coleta de dados para a análise do contexto ${ }^{10,11}$. Por fim, as inferências foram embasadas em evidências científicas prévias a esta pesquisa.

\section{Considerações finais}

Os fatores mais identificados no PMM como sendo influenciadores da formulação da política e concorrentes às Ideias (evidência científica) foram as Instituições (estrutura governamental e legado político), os Fatores Externos (eleições presidenciais) e os Interesses de diversos atores. Nos acordos bilaterais em Portugal, os entrevistados consideraram um processo influenciado predominantemente pelos fatores Interesses e Instituições (centralização do processo), com a restrição do papel da evidência para a entrada da problemática na agenda política.

Ao contrário do que se verificou no estudo de caso do Brasil, em que reconhecidamente a evidência teve um papel importante na formulação da política em análise, em Portugal a evidência científica gerada por pesquisa não foi identificada como contributo para a formulação da estratégia em estudo. No estudo, foram identificados diferentes determinantes para a restrição do papel da evidência na formulação da estratégia de contratação de médicos estrangeiros por acordos bilaterais e o uso da evidência no processo de formulação do PMM.

Por um lado, a característica do processo de formulação da política do país - o processo político português em análise foi centralizado em poucos atores; e no caso do Brasil, o processo envolveu diversos atores na sua formulação, entre os quais o Ministério da Saúde com a participação da Casa 
Civil e MEC, e também contou com espaços de discussões inerentes à caraterística do processo de formulação de políticas do país. Por outro, a cultura organizacional do uso da evidência - no Brasil, a evidência vem sendo incluída no processo de formulação com alguns instrumentos, nomeadamente a agenda de pesquisa e com estratégias como o ObservaRH. Em Portugal não foram reconhecidos mecanismos institucionais que promovessem o uso da evidência na formulação das políticas.

Nos dois países analisados foi identificada a necessidade de intervenções e políticas que considerem todas as áreas do mercado de trabalho em saúde e contenham ações que permeiem outros setores além da saúde. O potencial reforço de utilização da evidência científica na formulação de políticas poderia ser alcançado, nomeadamente, com: o investimento em ambientes de discussão e plataformas de interações entre investigadores, formuladores e outros usuários de resultados de investigação; e o aumento do apoio à investigação em termos de financiamento e mecanismos de estabelecimento de prioridades políticas.

É importante ressaltar a necessidade de futuras investigações que avaliem as estratégias implantadas nesses dois países; analisem o fluxo de saídas dos profissionais, principalmente emigração, saída para o setor privado ou outros setores e duplo emprego; facultem um diagnóstico mais preciso sobre a má distribuição dos médicos entre os níveis de atenção; explorem a cultura organizacional do uso de evidência analisando crenças e atitudes; analisem a atuação de organizações knowledge-brokering, utilizando outras políticas implementadas; e mais estudos acerca dos fatores que levariam os médicos a migrar e atuar em regiões desassistidas.

\section{Colaboradores}

A. P. C. Oliveira contribuiu com o desenho do estudo, coleta e análise dos dados, estruturação e escrita do artigo, e revisão da redação em todas as versões incluindo a final. M. R. Dal Poz, I. Craveiro, M. Gabriel e G. Dussault contribuíram com o desenho do estudo e revisão da redação do artigo em todas as versões incluindo a final.

\section{Agradecimentos}

Os autores agradecem ao Professor John N. Lavis, pelos valiosos comentários sobre o método estudo de caso-múltiplo; aos atores entrevistados, pelo seu tempo e valiosas informações prestadas; e ao Pedro Leite Alves pelo apoio técnico. Ao $\mathrm{CNPq}$ (bolsa de doutorado 01988/2012-7) e à Faperj (E26/201359/2014) pelo financiamento.

\section{Referências}

1. Oxman AD, Lavis JN, Lewin S, Fretheim A. SUPPORT Tools for evidence-informed health Policymaking (STP) 1: what is evidence-informed policymaking? Health Res Policy Syst 2009; 7 Suppl 1:S1.

2. Strydom WF, Funke N, Nienaber S, Nortje K, Steyn M. Evidence-based policymaking: a review. S Afr J Sci 2010; 106:1-8.

3. Campbell S, Benita S, Coates E, Davies P, Penn G. Analysis for policy: evidence-based policy in practice. http://preval.org/files/ pu256_160407.pdf (acessado em 30/Dez/ 2016).

4. Ward V, House A, Hamer S. Developing a framework for transferring knowledge into action: a thematic analysis of the literature. J Health Serv Res Policy 2009; 14:156-64.

5. Graham ID, Logan J, Harrison MB, Straus SE, Tetroe J, Caswell W, et al. Lost in knowledge translation: time for a map? J Contin Educ Health Prof 2006; 26:13-24.

6. World Health Organization. World report on knowledge for better health: strengthening health systems. Geneva: World Health Organization; 2004.

7. Oliver K, Innvar S, Lorenc T, Woodman J, Thomas J. A systematic review of barriers to and facilitators of the use of evidence by policymakers. BMC Health Serv Res 2014; 14:2. 
8. Innvaer S, Vist G, Trommald M, Oxman A. Health policy-makers' perceptions of their use of evidence: a systematic review. J Health Serv Res Policy 2002; 7:239-44.

9. Waddell C, Lavis JN, Abelson J, Lomas J, Shepherd CA, Bird-Gayson T, et al. Research use in children's mental health policy in Canada: maintaining vigilance amid ambiguity. Soc Sci Med 2005; 61:1649-57.

10. Oliveira APC, Gabriel M, Dal Poz MR, Dussault G. Desafios para assegurar a disponibilidade e acessibilidade à assistência médica no Sistema Único de Saúde. Ciênc Saúde Coletiva 2017; 22:1165-80.

11. Oliveira APC, Dussault G, Craveiro I. Challenges and strategies to improve the availability and geographic accessibility of physicians in Portugal. Hum Resour Health 2017; 15:24.

12. Lavis JN, Rottingen JA, Bosch-Capblanch X, Atun R, El-Jardali F, Gilson L, et al. Guidance for evidence-informed policies about health systems: linking guidance development to policy development. PLoS Med 2012; 9:e1001186.

13. Pomey M-P, Morgan S, Church J, Forest P-G, Lavis JN, McIntosh T, et al. Do provincial drug benefit initiatives create an effective policy lab? The evidence from Canada. J Health Polit Policy Law 2010; 35:705-42.

14. Oliveira JPA, Sanchez MN, Santos LMP. O Programa Mais Médicos: provimento de médicos em municípios brasileiros prioritários entre 2013 e 2014. Ciênc Saúde Coletiva 2016; 21:2719-27.

15. Chopra M, Munro S, Lavis JN, Vist G, Bennett S. Effects of policy options for human resources for health: an analysis of systematic reviews. Lancet 2008; 371:668-74.

16. World Health Organization. Transforming and scaling up health professionals' education and training: World Health Organization guidelines 2013. Geneva: World Health Organization; 2013.

17. World Health Organization. Increasing access to health workers in remote and rural areas through improved retention. Geneva: World Health Organization; 2010.

18. Hanney SR, Gonzalez-Block MA, Buxton MJ, Kogan M. The utilisation of health research in policy-making: concepts, examples and methods of assessment. Health Res Policy Syst 2003; $1: 2$.

19. Lavis JN. Research, public policymaking, and knowledge-translation processes: Canadian efforts to build bridges. J Contin Educ Health Prof 2006; 26:37-45.

20. Amara N, Ouimet M, Landry R. New evidence on instrumental, conceptual, and symbolic utilization of university research in government agencies. Sci Commun 2004; 26:75-106.

21. Trostle J, Bronfman M, Langer A. How do researchers influence decision-makers? Case studies of Mexican policies. Health Policy Plan 1999; 14:103-14.

22. Lavis JN, Lomas J, Hamid M, Sewankambo NK. Assessing country-level efforts to link research to action. Bull World Health Organ 2006; 84:620-8.
23. Carvalho CL, Petta HL, Araújo JF, Girardi Junior JB, Oliveira VA. Avaliação nacional da demanda de médicos especialistas percebida pelos gestores de saúde. Belo Horizonte: Núcleo de Estudos em Saúde Coletiva, Faculdade de Medicina, Universidade Federal de Minas Gerais; 2009

24. Carvalho CL, Farah JM, Araújo JF, Wan der Maas L, Campos LAB. Construção do índice de escassez de profissionais de saúde para apoio à Política Nacional de Promoção da Segurança Assistencial em Saúde. Belo Horizonte: Núcleo de Estudos em Saúde Coletiva, Faculdade de Medicina, Universidade Federal de Minas Gerais; 2010.

25. Observatório de Recursos Humanos em Saúde/Estação de Trabalho do IMS/UERJ. Sistema de Indicadores das Graduações em Saúde - SIGRAS. http://www.obsnetims.org. br/sigras/home.php (acessado em 30/Dez/ 2016).

26. Seixas PH, Corrêa A, Moraes J. Migramed - Migração Médica no Brasil: tendências e motivações. In: Pierantoni CR, Dal Poz MR, França T, organizadores. O trabalho em saúde: abordagens quantitativas e qualitativas. Rio de Janeiro: Centro de Estudos, Pesquisa e Desenvolvimento Tecnológico em Saúde Coletiva, Instituto de Medicina Social, Universidade do Estado do Rio de Janeiro/ObservaRH; 2011. p. 133-50.

27. Scheffer M, Biancarelli A, Cassenote A. Demografia médica no Brasil. v. 2: dados gerais e descrições de desigualdades. São Paulo: Conselho Regional de Medicina do Estado de São Paulo/Conselho Federal de Medicina; 2013.

28. Haynes AS, Gillespie JA, Derrick GE, Hall WD, Redman S, Chapman S, et al. Galvanizers, guides, champions, and shields: the many ways that policymakers use public health researchers. Milbank Q 2011; 89:564-98.

29. Greyson DL, Cunningham C, Morgan S. Information behaviour of Canadian pharmaceutical policy makers. Health Info Libr J 2012; 29: 16-27.

30. Grupo de Missão. Plano estratégico para a formação nas áreas da saúde. Lisboa: Grupo de Missão; 2001.

31 Santana P, Couceiro L, Alves I, Nogueira H, Costa C, Santos R. Caracterização demográfica dos profissionais de saúde no serviço nacional de saúde português. v. V: sumário executivo. Lisboa: Secretaria-Geral, Ministério da Saúde; 2007.

32. Santana P, Peixoto H, Loureiro A, Costa C, Nunes C, Duarte N. Estudo de evolução prospectiva de médicos no sistema nacional de saúde: relatório final. Coimbra: Ordem dos Médicos; 2013.

33. Dussault G. Plano nacional de saúde 20122016: roteiro de intervenção em recursos humanos em saúde (RHS). Lisboa: Direção-Geral da Saúde; 2014.

34. Ministério da Saúde. Programa Mais Médicos - dois anos: mais saúde para os brasileiros. Brasília: Ministério da Saúde; 2015. 
35. Oliveira FP, Vanni T, Pinto HA, Santos JTR, Figueiredo AM, Araújo SQ, et al. Mais Médicos: um programa brasileiro em uma perspectiva internacional. Interface (Botucatu, Online) 2015; 19:623-34.

\section{Abstract}

This study aims to analyze whether the process by which policies for human resources for health that aim to improve the geographic distribution of physicians have been informed by scientific evidence in Brazil and Portugal. This was a multiple case study on a decision-making process for human resources for health in Brazil and Portugal. The respective case studies were based on Brazil's More Doctors Program (Programa Mais Médi$\cos -P M M)$ and Portugal's strategy of hiring foreign physicians through bilateral agreements, to work in the country's National Health Service (SNS). We interviewed 27 key actors in the policy-making process on the following topics: factors that influenced the policy decisions, actors that were expected to win or lose from the policy, and the scientific evidence and available data used in the policy-making, among others. The most evident factors appearing in the interviews as having influenced the PMM were: institutions; external factors (Presidential elections); group interests (e.g. physicians' professional associations), governments (Brazil and Cuba), international organizations, and civil society; and ideas (scientific evidence). The most frequently cited factors in Portugal were: institutions and interests of government (from Portugal and the countries involved in the bilateral agreements), civil society, and groups (physicians' professional associations). Contrary to the case study in Brazil, where the evidence was reported to having played an important role in the policy decisions, in Portugal, scientific evidence was not identified as contributing to the specific policy process.

Health Manpower; Public Health Policy; Policy Making; Medically Underserved Area; Health Services Accessibility
36. Pierson P. When effect becomes cause: policy feedback and political change. World Polit 1993; 45:595-628.

\section{Resumen}

El estudio tiene por objetivo analizar el proceso en el que las políticas de recursos humanos en salud (RHS), que tienen como fin la mejora de la distribución geográfica de los médicos, son (o no) informadas por evidencias científicas en Brasil y en Portugal. Se trata de un estudio de caso-múltiple sobre el proceso de decisión de las politicas de RHS en Brasil y en Portugal. Para configurar los estudios de caso, las politicas elegidas fueron el Programa Más Médicos (PMM) y la estrategia de contratación de médicos extranjeros mediante acuerdos bilaterales para el trabajo en el Servicio Nacional de Salud (SNS) portugués. Se entrevistaron a 27 actores-clave en el proceso de formulación de las politicas en el análisis en los siguientes asuntos: factores que influenciaron la formulación, actores que se esperaba ganar o perder, evidencias científicas y datos disponibles utilizados para la formulación, entre otros. Los factores más evidentes, identificados en las entrevistas como de influencia en el PMM, fueron: instituciones; factores externos (elecciones presidenciales); intereses de grupos (por ejemplo, asociaciones de profesionales médicos), gobiernos (brasileño y cubano), organización internacional y sociedad civil; e ideas (evidencia científica). Los factores más registrados en Portugal fueron: instituciones e intereses de los gobiernos (como el portugués y los involucrados en los acuerdos bilaterales), sociedad civil y grupos (asociaciones de profesionales médicos). Al contrario de lo que se verificó en el estudio de caso de Brasil, donde se reconoció que la evidencia tuvo un papel importante en la formulación de la politica en análisis, en Portugal la evidencia científica no fue identificada como una contribución para la formulación de la intervención en estudio.

Recursos Humanos en Salud; Politicas Públicas de Salud; Formulación de Politicas; Área sin Atención Médica; Accesibilidad a los Servicios de Salud

Recebido em 30/Dez/2016

Versão final reapresentada em 30/Jun/2017 Aprovado em 17/Jul/2017 